\title{
ON COMPLEX HOMOGENEOUS SPACE OF VECTORS WITH CONSTRAINTS
}

\author{
EMILIJA CELAKOSKA, ELENA HADZIEVA AND VESNA CELAKOSKA- \\ JORDANOVA
}

Communicated by Ivaïlo Mladenov

Abstract. A homogeneous space $\mathcal{V}$ of complex constrained vectors in $\mathbb{C}^{3}$, representing complex velocities is introduced. The corresponding representation of the complex special orthogonal group of transformations acting on $\mathcal{V}$ is also examined. The requirement for real vector magnitudes is addressed by imposing orthogonality between the real and the imaginary parts of vectors and use of the non-conjugate scalar product. We present the orthogonal transformations acting on $\mathcal{V}$ in terms of the polar decomposition of complex orthogonal matrices. The group link problem and the homogeneity of the space $\mathcal{V}$ are also discussed. Finally, we briefly consider the convenience of the space $\mathcal{V}$ in theoretical calculations.

MSC: 51F25, 32M05, 20C35, 83D05

Keywords: Boost, complex orthogonal group, group link, group representation

\section{Introduction}

It is a common knowledge in the Lie group theory that the complex rotation group $\mathrm{SO}(3, \mathbb{C})$ is isomorphic to the restricted Lorentz group $\mathrm{SO}^{+}(1,3)$. Due to this isomorphism, the group $\mathrm{SO}(3, \mathbb{C})$ is sometimes an alternative choice to represent particular aspects of some physical theories ordinarily expressed in terms of $\mathrm{SO}^{+}(1,3)$. In regard to physical applications, it is important to outline some of the useful properties of the complex space $\mathbb{C}^{3}$ compared to commonly used fourdimensional real space: it supports the use of vector product, the increased number of vector components can be related to additional physical quantities and it offers a possibility to discern some commonly intertwined physical concepts by employing the real/imaginary separation. As examples of using $\mathrm{SO}(3, \mathbb{C})$ in physical applications one can see [1], [2], [10], [14] among others. It is noteworthy to remark that the representation of the group $\operatorname{SO}(3, \mathbb{C})$ therein is adapted to the standard unconstrained vectors in $\mathbb{C}^{3}$. When the $\mathrm{SO}(3, \mathbb{C})$ transformation is parameterized by a vector of velocity, it is commonly taken to be a three-dimensional vector $\vec{v}$ with real components. Even when the vector of velocity is actually a complex vector 
like in the bi-quaternion (quaternions with complex parameters) models of relativity [14], the corresponding representation of the group $\mathrm{SO}(3, \mathbb{C})$ has not been specifically addressed.

In this paper, we introduce a homogenous space $\mathcal{V}$ of complex constrained vectors in $\mathbb{C}^{3}$, representing complex velocities. The requirement for real vector magnitudes is addressed by imposing orthogonality between the real and the imaginary parts of vectors and adopting the non-conjugate scalar product. The standard alternative, to use the conjugate scalar product, does not provide "hyperbolic" calculation of vector magnitudes.

Our aim is to examine the representation of $\mathrm{SO}(3, \mathbb{C})$ transformations which act on $\mathcal{V}$. Apart from the existing approaches mentioned above which exploit the common representation of $\mathrm{SO}(3, \mathbb{C})$, we adapt the representation of the group $\mathrm{SO}(3, \mathbb{C})$ to agree with the prescribed constraints on the complex vectors in $\mathcal{V}$. The standard polar decomposition of complex orthogonal matrices on (real orthogonal)/(positive definite Hermitian) corresponds to the rotation/boost decomposition of matrices representing transformations acting on $\mathcal{V}$. We focus our attention on boost representation in $\mathcal{V}$ which correspondingly differs from the standard boost representation in $\mathrm{SO}(3, \mathbb{C})$ acting on $\mathbb{C}^{3}$. The homogeneity of the space $\mathcal{V}$ is examined through the transitivity of the boost action. Additionally, we examine a group link between vectors in $\mathcal{V}$ which contains a local boost action, where the boost preserves the direction of the real part of the vector. An adaptation of $\mathcal{V}$ toward a space of vector potentials and its simple geometrization is also briefly discussed.

\section{The Space of Complex Constrained Vectors}

We consider the ordinary non-conjugate scalar product $\cdot$ in $\mathbb{C}^{3}$ such that $\left(\mathbb{C}^{3}, \cdot\right)$ is complex inner space with weakened scalar product conditions. Of course, this scalar product in general, does not provide real vector magnitudes.

Now, let us consider a subset $\mathcal{V} \subset \mathbb{C}^{3}$ consisting of complex vectors $v \in \mathbb{C}^{3}$ of the form

$$
v=\vec{x}+\mathrm{i} \vec{y}, \quad \vec{x}, \vec{y} \in \mathbb{R}^{3}
$$

with constraints $\vec{x} \vec{y}=0$ and $v \cdot v=c^{2}$, for constant $c, 0<c \in \mathbb{R}$. We will use equivalent and physically more relevant description of the space $\mathcal{V}$ given by the following definition.

Definition 1. The space $\mathcal{V} \subset \mathbb{C}^{3}$ is defined by

$$
\mathcal{V}=\left\{v=\gamma_{v}(c \vec{n}+\mathrm{i} \vec{v}) ;\|\vec{n}\|=1, \vec{n} \vec{v}=0\right\}
$$

where $\gamma_{v}=1 / \sqrt{1-\vec{v}^{2} / c^{2}}$ is the Lorentzfactor. 
The magnitude of the vector $v$ is calculated regarding the scalar product , i.e., $v \cdot v=c^{2}$. The scalar product $\cdot$ is obviously complex valued scalar product in $\mathcal{V}$ where the orthogonality constraint $\vec{n} \vec{v}=0$ yields real vector magnitudes.

Notice that vector addition and multiplication with scalar inherited from $\mathbb{C}^{3}$ are not closed operations in $\mathcal{V}$ and so, $\mathcal{V}$ is not a vector subspace of $\mathbb{C}^{3} . \mathcal{V}$ would not be a vector space even if vector addition and multiplication by a scalar were closed operations, introduced by using the corresponding boost transformations given in the next section. In that case, the vector addition would be a non-commutative, non-associative and also a non-distributive operation with regard to scalar multiplication. These observations are comparable to the four-vectors of velocity in Minkowski space. However, in the latter, the time component depends on the space components and thus the corresponding homogeneous space is three-dimensional, while $\operatorname{dim}(\mathcal{V})=4$ (with respect to $\mathbb{R}$ ) because of the conditions $\vec{n} \vec{v}=0$ and $\|\vec{n}\|=1$ which reduce two dimensions down from six. The condition $v \cdot v=c^{2}$ does not reduce dimensionality since this condition follows from the previous ones and the presence of the scalar $\gamma_{v}$. So, the homogeneous space $\mathcal{V}$ is not isomorphic to the homogeneous space of four-vectors of velocity in Minkowski space because of the difference in dimensionality. Actually, it is obvious that $\mathcal{V}$ can be naturally mapped onto the homogeneous space of four-vectors of velocity by the surjection $\gamma_{v}(c \vec{n}+\mathrm{i} \vec{v}) \mapsto \gamma_{v}(c, \vec{v})$.

The various disadvantages of the scalar product . in $\mathcal{V}$ are compensated by the "hyperbolic" calculation of the vector magnitudes. The standard alternative is to use the conjugate scalar product which is common in complex spaces. However, in such case, $\mathcal{V}$ could not be placed in parallel with the homogeneous space of four-vectors of velocity in Minkowski space. Observe that using "imaginary" $\vec{v}$ in the representation of the complex vectors in $\mathcal{V}$ corresponds to the diagonal metric $\operatorname{diag}(1,-1,-1,-1)$ in the Minkowski space.

The vectors $v=\gamma_{v}(c \vec{n}+\mathrm{i} \vec{v})$ in $\mathcal{V}$ allow the following physical interpretation. The imaginary part $\gamma_{v} \vec{v}$ can be interpreted as the relativistic velocity known from the Minkowski space. The real part given by $\gamma_{v} c \vec{n}$ could be associated with an observer, where $\vec{n}$, as a unit vector, could represent the direction of the observer's time velocity. In that case, $\vec{n}$ should be a dimensionless quantity (time/time). The transformations presented in the next section vary the velocity given in the imaginary part of vectors, but they also change the direction of the observer's time velocity. 


\section{Transformations in $\mathcal{V}$}

The $\mathrm{SO}(3, \mathbb{C})$ isomorphism with $\mathrm{SO}^{+}(1,3)$ means that there are corresponding counterparts of the elements of $\mathrm{SO}^{+}(1,3)$ in $\mathrm{SO}(3, \mathbb{C})$. However, we are interested in representation $G$ of the group $\mathrm{SO}(3, \mathbb{C})$ acting on the space $\mathcal{V}$.

It is well-known that a matrix $A$ is complex orthogonal $\left(A^{\top} A=I\right)$ if and only if there is a real orthogonal matrix $R$ and a real antisymmetric matrix $S$ such that $A=R \mathrm{e}^{\mathrm{i} S}$, (see for example [6, p.487]). In this polar decomposition, the matrix $\mathrm{e}^{\mathrm{i} S}$ is a positive definite Hermitian coninvolutory matrix. Of course, the rotation/boost decomposition of the matrices in $G$ should correspond to the polar decomposition of orthogonal complex matrices. In light of this, we can simply recognize that the rotations in $G$ are reduced to the ordinary $\mathrm{SO}(3)$ rotations. Usually, they are represented by the Rodrigues' rotation formula

$$
R_{\vec{s}, \theta}=\cos \theta I+\sin \theta[\vec{s}]^{\times}+(1-\cos \theta) \vec{s} \otimes \vec{s}
$$

where $I$ is the identity matrix, $\vec{s}$ is a unit vector in direction of the rotation axis, $\theta$ is the angle of rotation and $[\vec{s}]^{\times}$is the cross product matrix of $\vec{s}$ (see e.g., [8, p.281]). Let $u=\gamma_{u}(c \vec{m}+\mathrm{i} \vec{u})$ and $v=\gamma_{v}(c \vec{n}+\mathrm{i} \vec{v})$ be two vectors in $\mathcal{V}$. Sometimes, of particular interest will be the rotation that brings the unit vector $\vec{m}$ to the unit vector $\vec{n}$. We can choose the rotation axis to be orthogonal to both $\vec{m}$ and $\vec{n}$ and the Rodrigues' rotation formula adapted to this specific case takes the form

$$
R_{\vec{n}}^{\vec{m}}=\vec{m} \vec{n} I+[\vec{n} \times \vec{m}]^{\times}+\frac{1}{1+\vec{m} \vec{n}}(\vec{n} \times \vec{m}) \otimes(\vec{n} \times \vec{m}) .
$$

Of course, the boosts are fundamental transformations in $G$, because unlike rotations, they vary the magnitudes of the real and the imaginary parts of the vectors.

Definition 2. A boost $B_{v}$ in $G$ parameterized by a vector $v=\gamma_{v}(c \vec{n}+\mathrm{i} \vec{v}) \in \mathcal{V}$ is represented by the following matrix

$$
B_{v}=I+\left(\gamma_{v}-1\right) \vec{n} \otimes \vec{n}+\frac{\gamma_{v}^{2}}{\left(1+\gamma_{v}\right) c^{2}} \vec{v} \otimes \vec{v}+\mathrm{i} \frac{\gamma_{v}}{c}[\vec{n} \times \vec{v}]^{\times} .
$$

In order $B_{v}$ to correspond to the polar decomposition of complex orthogonal matrices, it should be orthogonal and positive definite Hermitian coninvolutory matrix. There is a comparable situation in Minkowski space, where it is shown that rotation/boost decomposition of $\mathrm{SO}^{+}(1,3)$ matrices is exactly the polar decomposition of real orthogonal matrices [12, Ch.5]. The boost representation (1) could be put in parallel to the Lorentz boost in general frame [5, p.197]. 
Proposition 3. The boost $B_{v}$, as given by (1), is an orthogonal positive definite Hermitian coninvolutory matrix.

Proof: It is easy to directly obtain $\Re\left(B_{v}\right) \Im\left(B_{v}\right)=\Im\left(B_{v}\right) \Re\left(B_{v}\right)$ and so

$$
B_{v} B_{\bar{v}}=\left(I+\left(\gamma_{v}-1\right) \vec{n} \otimes \vec{n}+\frac{\gamma_{v}^{2}}{\left(1+\gamma_{v}\right) c^{2}} \vec{v} \otimes \vec{v}\right)^{2}+\left(\frac{\gamma_{v}}{c}[\vec{n} \times \vec{v}]^{\times}\right)^{2}=I=B_{\bar{v}} B_{v} .
$$

This gives $B_{\bar{v}}=B_{v}^{-1}$, but we also have $B_{\bar{v}}=B_{v}^{\top}$, so $B_{v}$ is orthogonal. Obviously, $\bar{B}_{v}=B_{v}^{\top}$, so $\bar{B}_{v}^{\top}=B_{v}$ and $B_{v} \bar{B}_{v}=I$, that is, $B_{v}$ is a Hermitian coninvolutory matrix. From the straightforward calculation of $B_{v} u$ where $u=\gamma_{u}(c \vec{m}+\mathrm{i} \vec{u}) \in \mathcal{V}$,

$$
\begin{aligned}
B_{v} u= & c\left(\gamma_{u} \vec{m}+\left(\gamma_{u}\left(\gamma_{v}-1\right) \vec{m} \vec{n}+\frac{\gamma_{u} \gamma_{v}}{c^{2}} \vec{u} \vec{v}\right) \vec{n}+\left(\frac{\gamma_{v}{ }^{2} \gamma_{u}}{\left(1+\gamma_{v}\right) c^{2}} \vec{m} \vec{v}-\frac{\gamma_{v} \gamma_{u}}{c^{2}} \vec{n} \vec{u}\right) \vec{v}\right) \\
& +\mathrm{i}\left(\gamma_{u} \vec{u}+\left(\gamma_{u}\left(\gamma_{v}-1\right) \vec{n} \vec{u}-\gamma_{v} \gamma_{u} \vec{m} \vec{v}\right) \vec{n}+\left(\frac{\gamma_{v}^{2} \gamma_{u}}{\left(1+\gamma_{v}\right) c^{2}} \vec{u} \vec{v}+\gamma_{v} \gamma_{u} \vec{m} \vec{n}\right) \vec{v}\right)
\end{aligned}
$$

it can be obtained that $\bar{u}\left(B_{v} u\right)>0$. Namely,

$$
\begin{aligned}
\bar{u}\left(B_{v} u\right)= & \left(\gamma_{u} \sqrt{\gamma_{v}-1} \vec{n} \vec{u}-\frac{\gamma_{u} \gamma_{v}}{\sqrt{1+\gamma_{v}}} \vec{m} \vec{v}\right)^{2}+\left(c \gamma_{u} \sqrt{\gamma_{v}-1} \vec{m} \vec{n}+\frac{\gamma_{u} \gamma_{v}}{\sqrt{c\left(1+\gamma_{v}\right)}} \vec{u} \vec{v}\right)^{2} \\
& +\gamma_{u}^{2}\left(c^{2}+\vec{u}^{2}\right)+2 \gamma_{u}^{2} \gamma_{v}\left(1-\sqrt{\frac{\gamma_{v}-1}{\gamma_{v}+1}}\right)((\vec{m} \vec{n})(\vec{u} \vec{v})-(\vec{m} \vec{v})(\vec{n} \vec{u})) .
\end{aligned}
$$

Now, if $(\vec{m} \vec{n})(\vec{u} \vec{v})-(\vec{m} \vec{v})(\vec{n} \vec{u})>0$, then obviously $\bar{u}\left(B_{v} u\right)>0$. On the other hand, if $(\vec{m} \vec{n})(\vec{u} \vec{v})-(\vec{m} \vec{v})(\vec{n} \vec{u})<0$, we use the fact that $\gamma_{v}\left(1-\sqrt{\frac{\gamma_{v}-1}{\gamma_{v}+1}}\right)<1$ and $(\vec{m} \vec{n})(\vec{u} \vec{v})-(\vec{m} \vec{v})(\vec{n} \vec{u})=(\vec{m} \times \vec{u})(\vec{n} \times \vec{v})>-\|\vec{u}\|\|\vec{v}\|$, and obtain

$$
\begin{aligned}
\gamma_{u}^{2}\left(c^{2}+\vec{u}^{2}\right)+2 \gamma_{u}^{2} \gamma_{v}\left(1-\sqrt{\frac{\gamma_{v}-1}{\gamma_{v}+1}}\right)((\vec{m} \vec{n})(\vec{u} \vec{v})-(\vec{m} \vec{v})(\vec{n} \vec{u})) \\
>\gamma_{u}^{2}\left(\vec{v}^{2}+\vec{u}^{2}\right)-2 \gamma_{u}^{2}\|\vec{u}\|\|\vec{v}\|=\gamma_{u}^{2}(\|\vec{u}\|-\|\vec{v}\|)^{2}>0 .
\end{aligned}
$$

The action of the matrix $B_{v}$ on vectors in $\mathcal{V}$ is comparable with the characteristic manner of boost action in Minkowski space. Namely, if $B_{v}$ acts on a "zero" vector $c \vec{n}$, the result is $v, B_{v} c \vec{n}=v$, and if it acts on a vector $u=\gamma_{u}(c \vec{n}+\mathrm{i} \vec{u})$ with the same real part direction as $v$, the result is

$$
x=B_{v} u=\gamma_{u} \gamma_{v} c\left(1+\frac{\vec{u} \vec{v}}{c^{2}}\right) \vec{n}+\mathrm{i}\left(\gamma_{v} \vec{u}+\gamma_{u} \gamma_{v} \vec{v}+\frac{\gamma_{u}^{2} \gamma_{v}}{\left(1+\gamma_{u}\right) c^{2}}(\vec{u} \vec{v}) \vec{v}\right)
$$

where $\Im(x) /(\Re(x) \vec{n})$ is actually the relativistic velocity addition. Also, it is easy to confirm that two boosts, in general case, do not commute, $B_{u} B_{v} \neq B_{v} B_{u}$, even 
in cases when they are generated from vectors with a common direction of their real parts. The product $B_{u} B_{v}$ is not a Hermitian matrix generally, thus, a product of boosts does not give a boost.

Given a complex matrix $B_{v}$ representing a boost in $\mathcal{V}$, there is no simple formula to extract the vector $v$ parameterizing $B_{v}$. However, one way to obtain the vector $v$ is to consider a system of equations using convenient matrix elements. The sum of diagonal elements in $B_{v}$ straightforwardly gives the solution for $\gamma_{v}$. Then, the imaginary parts of the matrix elements in the upper right (or the lower left) triangle give the components of $\vec{n} \times \vec{v}$. So, one can express the components of $\vec{v}$ linearly in the components of $\vec{n}$ using the equality $(\vec{n} \times \vec{v}) \times \vec{n}=\vec{v}$ which follows from the constraints. Finally, the components of $\vec{v}$ can be substituted in the real parts of elements in the upper right (or the lower left) triangle and the diagonal elements, in order to obtain a system of equations which gives the components of the vector $\vec{n}$.

It is interesting to compare the boost representation (1) acting on $\mathcal{V}$ with the standard boost representation in $\mathrm{SO}(3, \mathbb{C})$ acting on $\mathbb{C}^{3}$. In the local isomorphism $\mathrm{SO}^{+}(1,3) \rightarrow \mathrm{SO}(3, \mathbb{C})$ the vector parameterizing the boost is actually a real vector $\vec{v}$, and the corresponding boost representation in $\mathrm{SO}(3, \mathbb{C})$ acting on $\mathbb{C}^{3}$ takes the form

$$
B_{\vec{v}}=\gamma_{v} I+\frac{\gamma_{v}^{2}}{\left(1+\gamma_{v}\right) c^{2}} \vec{v} \otimes \vec{v}+\mathrm{i} \frac{\gamma_{v}}{c}[\vec{v}]^{\times} .
$$

Compare (4) with our boost representation given by (1) where the boost is parameterized by a complex vector $v$. Observe that in (2), the $\Re\left(B_{v} u\right)$ is a linear combination of $\vec{m}, \vec{n}, \vec{v}$ while the $\Im\left(B_{v} u\right)$ is a linear combination of $\vec{u}, \vec{n}, \vec{v}$. Compared to $B_{\vec{v}} u$, the presence of $\vec{n}$ in $B_{v} u$ obviously results in more "organized" boosting of the real and the imaginary part of $u$.

\section{G-Links Between Vectors in $\mathcal{V}$}

Let $u$ and $v$ be vectors in $\mathcal{V}$. Then, a $G$-link between $u$ and $v$ is a transformation $T$ in $G$ that carries $u$ to $v$, i.e., $T u=v$. We will show that $G$ acts on $\mathcal{V}$ transitively, namely, for each pair of vectors $u, v \in \mathcal{V}$ there is a $G$-link.

Proposition 4. $\mathcal{V}$ is a homogeneous space.

Proof. Let $u$ and $v$ be two vectors in $\mathcal{V}$. Since the scalar product is non-conjugate, analogously to the boost link in Minkowski space (see e.g. [3], [13], [11, Ch.11]), 
the standard boost $G$-link from $u$ to $v$ is given by

$$
B_{v}^{u}=I-\frac{2}{(u+v)^{2}}(u+v) \otimes(u+v)+\frac{2}{c^{2}} v \otimes u, \quad(u+v)^{2} \neq 0
$$

where the addition of vectors is inherited from $\mathbb{C}^{3}$.

Since $(u+v) \cdot u=c^{2}+u \cdot v$ and $(u+v)^{2}=2\left(c^{2}+u \cdot v\right)$, one can obtain orthogonality and $B_{v}^{u} u=v$ using direct calculations.

The boost definition in $\mathcal{V}$ given by (1) can be examined more generally in light of the group link problem. Namely, given a space $\mathcal{X}$ with a group $G$ acting transitively on $\mathcal{X}$, the problem is to determine a transformation $T_{y}^{x} \in G$ such that $T_{y}^{x} x=y$ for a given pair $x, y \in \mathcal{X}$. The transformation $T_{y}^{x} \in G$ for which $T_{x}^{x}=I \in G$ is called a boost [7, p.805]. So, this condition could be used to define a boost in the homogenous space $\mathcal{X}$. In our case, one can easily show that in (5), $B_{u}^{u}=I$, and so $B_{v}^{u}$ is a boost. However, it is not a common approach to represent the boost through a boost $G$-link $B_{v}^{u}$ since such representation is in a basis containing the vector $u$, while in the standard boost representation (1) the boost matrix refers only to a vector $v$ determined in a basis containing a "zero" vector. Thus, to obtain a standard boost representation we can simply put $u=c \vec{n}$ in (5) which gives $B_{v}^{c \vec{n}}=B_{v}$. Thus, the boost $B_{v}$ given by (1) is a boost $G$-link (5) between a "zero" vector and $v$.

Let us determine the stabilizer of a point in $\mathcal{V}$. Since the action of $G$ is transitive, one can determine the stabilizer of a point using any convenient element in $\mathcal{V}$. The stabilizer of one of "zero" elements, say $c \vec{n} \in \mathcal{V}$, obviously excludes boosts. Therefore, it is composed of rotations that keep the vector $\vec{n}$ in place, i.e., rotations around the axis defined by $\vec{n}$. So, the stabilizer of a point is isomorphic to $\operatorname{SO}(2)$.

In some applications of particular interest can be a boost that performs a local action. It means that the boost will keep the direction of the real part of an arriving vector fixed. Let $B_{v}$ be a boost parameterized by $v=\gamma_{v}(c \vec{n}+\mathrm{i} \vec{v})$ and

$$
\mathcal{V}_{\vec{p}}=\left\{\gamma_{w}(c \vec{p}+\mathrm{i} \vec{w}) \in \mathcal{V} ; \vec{p} \text { is fixed }\right\} \subset \mathcal{V}
$$

is a set of vectors with the fixed direction $\vec{p}$ of their real parts. In order to preserve the direction of the real part of arriving vectors from $\mathcal{V}_{\vec{p}}$, we perform similarity transformation of the boost $B_{v}$ using the corresponding rotation matrix $R_{\vec{n}}^{\vec{p}}$ (rotation from $\vec{p}$ to $\vec{n}$ ).

Definition 5. The local boost action $\left(B_{v}\right)_{\vec{p}}: \mathcal{V}_{\vec{p}} \rightarrow \mathcal{V}_{\vec{p}}$ is represented by the following matrix

$$
\left(B_{v}\right)_{\vec{p}}=\left(R_{\vec{n}}^{\vec{p}}\right)^{-1} B_{v} R_{\vec{n}}^{\vec{p}}
$$


The matrix $\left(B_{v}\right)_{\vec{p}}$ is obviously orthogonal. It is easy to show that $u \cdot v>0$ for $u, v \in \mathcal{V}_{\vec{p}}$, which implies that $\left(B_{v}\right)_{\vec{p}}$ is a positive definite matrix on vectors in $\mathcal{V}_{\vec{p}}$. It is also Hermitian matrix since $B_{v}$ is Hermitian, and $R_{\vec{n}}^{\vec{p}}$ and its inverse $R_{\vec{p}}^{\vec{n}}$ are unitary matrices. Finally, $\left(B_{v}\right)_{\vec{p}}$ obviously transforms vectors from $\mathcal{V}_{\vec{p}}$ to $\mathcal{V}_{\vec{p}}$ by keeping the directions of their real parts.

Now, it is of interest to represent a boost $G$-link (5) in a way that the local boost action is explicitly expressed. So, we will define a $G$-link $T_{v}^{u}$ between $u$ and $v$ which is based on the local boost action. Whenever $\|\vec{u}\| \neq\|\vec{v}\|, T_{v}^{u}$ must contain a local boost action and when $\vec{m} \neq \vec{n}$ it must also contain a rotation.

Proposition 6. A boost $G$-link between $u$ and $v$ expressed by a local boost action can be given by the following transformation

$$
T_{v}^{u}=R_{\vec{n}}^{\vec{m}}\left(B_{R_{\vec{m}}^{\vec{n}} v}^{u}\right)_{\vec{m}}=R_{\vec{n}}^{\vec{m}} B_{R_{\vec{m}}^{\vec{n}} v}^{u}, \quad\left(u+R_{\vec{m}}^{\vec{n}} v\right)^{2} \neq 0
$$

where the transformation $B_{R_{\vec{m}}^{\vec{n}}}^{u}$ is given by the formula

$$
B_{R_{\vec{m}}^{\vec{n}} v}^{u}=I-\frac{2}{\left(u+R_{\vec{m}}^{\vec{n}} v\right)^{2}}\left(u+R_{\vec{m}}^{\vec{n}} v\right) \otimes\left(u+R_{\vec{m}}^{\vec{n}} v\right)+\frac{2}{c^{2}} R_{\vec{m}}^{\vec{n}} v \otimes u .
$$

Proof: Firstly, observe that $\left(B_{R_{\vec{m}}^{n} v}^{u}\right)_{\vec{m}}=B_{R_{m}^{\vec{n}} v}^{u}$ since the boost $B_{R_{\vec{m}}^{\vec{n}} v}^{u}$ is generated by a vector with the direction $\vec{m}$ of its real part. Thus, the rotation matrices in the corresponding similarity transformation are reduced to $I$. By straightforward calculations it is easy to confirm that (7) is orthogonal and also it is simple to check that the equality $B_{R_{\vec{m}}^{\vec{n}} v}^{u} u=R_{\vec{m}}^{\vec{n}} v$ is valid. Actually, according to (5), the equation (7) represents a boost G-link between $u$ and $R_{\vec{m}}^{\vec{n}} v$. Now, $T_{v}^{u}$ is obviously an orthogonal transformation and $T_{v}^{u} u=v$. Finally, since $T_{u}^{u}=I$, it follows that $T_{v}^{u}$ is a boost.

The local boost action corresponds to the boost action in the group $\mathrm{SO}(1,2)$ which is known to have applications in different branches of physics including classical, relativistic and particle mechanics.

\section{Discussion}

It is a straightforward task to extend the homogeneous space of velocities $\mathcal{V}$ by including a scalar potential $\varphi$ obtaining a homogeneous space $\mathcal{P} \subset \mathbb{C}^{3}$ of vector potentials. Let the space $\mathcal{P} \subset \mathbb{C}^{3}$ of vector potentials be defined by

$$
\mathcal{P}=\left\{p=-\frac{1}{c^{2}} \mathrm{i} \varphi v ; \varphi=\varphi(\vec{r}), v \in \mathcal{V}\right\}
$$


where $\varphi$ is a smooth function of position representing a scalar potential.

The vector potential $p$ corresponds to the concept of four-potential used in electromagnetism, gravitation [4] and some other field theories. Observe that the representation of $\operatorname{SO}(3, \mathbb{C})$ acting on $\mathcal{P}$ remains $G$ since $\varphi$ is a scalar field.

Now, we address the simplest case when the vectors in $\mathcal{P}$ have a static orthogonal component. Thus, let $\mathcal{P}_{\vec{n}}=\left\{p=\frac{\varphi}{c^{2}} \gamma_{v}(\vec{v}-\mathrm{i} c \vec{n})\right\} \subset \mathcal{P}$ is a set of vectors in $\mathcal{P}$ with a fixed direction $\vec{n}$ of their orthogonal components and $G(1,2) \subset G$ is a representation of the subgroup $\mathrm{SO}(1,2) \subset \mathrm{SO}(3, \mathbb{C})$ acting on $\mathcal{P}_{\vec{n}}$. Since $\mathcal{P}_{\vec{n}}$ is a homogeneous space, there is an identification $\mathcal{P}_{\vec{n}} \sim G(1,2) / G_{p}(1,2)$ where $G_{p}(1,2)$ is the stabilizer of an element $p \in \mathcal{P}_{\vec{n}}$. Now, the transport of the vector $q \in \mathcal{P}_{\vec{n}}$ can be naturally based on $\alpha=[\nabla \times p]^{\times}$(cross product matrix of the curl of $p$ ), where without loss of generality, for some $\varphi$ we can choose $p$ to correspond to a "zero" vector in $\mathcal{P}_{\vec{n}}$, i.e., $p=-\mathrm{i} \frac{\varphi}{c} \vec{n}$. Notice that the antisymmetric matrix $\alpha$ is an element of the Lie algebra at $p$.

The idea is to define the transport of $q$ by an adjoint representation $\omega=B_{p}^{q} \alpha B_{q}^{p}$ of $\alpha$, where $B_{q}^{p}$ is a boost link between $p$ and $q$. The matrix $\omega$ can be considered as a connection form on the space $\mathcal{P}_{\vec{n}}$ and could be used to derive equations of motion. The existence of boosts $B_{p}^{q}$ and $B_{q}^{p}$ around $\alpha$ is an indicator of the interdependence between mass and velocity. In electromagnetism they vanish since the particle charge is independent of velocity. The similar idea, although in Minkowski space, is already exploited elsewhere [9]. Indeed, as a future research, it is of interest to examine the behavior of $\omega$ in the theoretical calculations of some relativistic effects.

\section{Conclusion}

We examined a representation $G$ of the group $\mathrm{SO}(3, \mathbb{C})$ applied to the homogeneous space $\mathcal{V}$ of complex constrained vectors in $\mathbb{C}^{3}$, representing complex velocities. The rotation/boost decomposition of the matrices in $G$ corresponds to the polar decomposition of complex orthogonal matrices on (real orthogonal)/(positive definite Hermitian). Real vector magnitudes in $\mathcal{V}$ are provided by orthogonality between the real and the imaginary parts of vectors and employing the non-conjugate scalar product. The discussion is mainly focused on the boosts and their action on the space $\mathcal{V}$. We discuss the boost link in $\mathcal{V}$ and give a group link representation where the involved boost preserves the direction of the real part of vectors. Due to the correspondence with boost in $\mathrm{SO}(1,2)$, the local boost action can be used 
as a transformation related to object motion in various physical applications. Finally, we briefly discuss a simple modification of the space $\mathcal{V}$ in order to obtain its geometrization which can be convenient in theoretical calculations.

\section{References}

[1] Aste A., Complex Representation Theory of the Electromagnetic Field, J. Geom. Symmetry Phys. 28 (2012) 47-58.

[2] Delphenich D., A More Direct Representation for Complex Relativity, Ann. Phys. 16 (2007) 615-639.

[3] Fahnline D., A Covariant Four-Dimensional Expression for Lorentz Transformation, Am. J. Phys. 50 (1982) 818-821.

[4] Fedosin S., Energy, Momentum, Mass, and Velocity of a Moving Body in the Light of Gravitomagnetic Theory, Can. J. Phys. 92 (2014) 1-8.

[5] Gourgoulhon E., Special Relativity in General Frames, Springer, Berlin 2013.

[6] Horn A. and Johnson C., Topics in Matrix Analysis, Cambridge University Press, Cambridge 1991.

[7] Oziewicz Z., Isometry From Reflections Versus Isometry from Bivector, Adv. Appl. Clifford Algebras 19 (2009) 793-817.

[8] Rao S., The Rotation and Lorentz Groups and Their Representations for Physics, John Wiley \& Sons, New York 1989.

[9] Trenčevski K., Celakoska E. and Balan V., Research of Gravitation in Flat Minkowski Space, Int. J. Theor. Phys. 50 (2011) 1-26.

[10] Trenčevski K., On the Geometry of the Space-Time and Motion of the Spinning Bodies, Centr. Eur. J. Phys. 11 (2013) 296-316.

[11] Ungar A., Beyond the Einstein Addition Law and its Gyroscopic Thomas Precession: The Theory of Gyrogroups and Gyrovector Spaces, Kluwer Academic, Boston 2001.

[12] Urbantke H., Lorentz Transformations From Reflections: Some Applications, Found. Phys. Lett. 16 (2003) 111-117.

[13] van Wyk C., Lorentz Transformastions in Terms of Initial and Final Vectors, J. Math. Phys. 27 (1986) 1306-1314.

[14] Yefremov A., Physical Theories in Hypercomplex Geometric Description, Int. J. Geom. Methods Mod. Phys. 11 (2014) 1450062. 
Emilija Celakoska

Ss. Cyril and Methodius University Skopje

Department of Mathematics and Informatics

Faculty of Mechanical Engineering

Karpos II bb, 1000 Skopje, R. Macedonia

E-mail address: emilija.celakoska@mf.edu.mk

Elena Hadzieva

Faculty of Information Systems, Visualization, Multimedia and Animation University of Information Science and Technology "St. Paul the Apostle" Partizanska bb, 6000 Ohrid, R. Macedonia

E-mail address: elena.hadzieva@uist.edu.mk

Vesna Celakoska-Jordanova

Ss. Cyril and Methodius University Skopje

Institute of Mathematics

Faculty of Natural Sciences and Mathematics

Arhimedova 3, 1000 Skopje, R. Macedonia

E-mail address: vesnac jepmf.ukim.mk 